\title{
Low-cost Position and Force Measurement System for Payload Transport Using UAVs
}

\author{
Daniel Ceferino Gandolfo Claudio D. Rosales Lucio R. Salinas \\ J. Gimenez Ricardo Carelli \\ Institute of Automation (INAUT), National University of San Juan - CONICET, San Juan J5400ARL, Argentina
}

\begin{abstract}
In recent years, multiple applications have emerged in the area of payload transport using unmanned aerial vehicles (UAVs). This has attracted considerable interest among the scientific community, especially the cases involving one or several rotarywing UAVs. In this context, this work proposes a novel measurement system which can estimate the payload position and the force exerted by it on the UAV. This measurement system is low cost, easy to implement, and can be used either in indoor or outdoor environments (no sensorized laboratory is needed). The measurement system is validated statically and dynamically. In the first test, the estimations obtained by the system are compared with measurements produced by high-precision devices. In the second test, the system is used in real experiments to compare its performance with the ones obtained using known procedures. These experiments allowed to draw interesting conclusions on which future research can be based.
\end{abstract}

Keywords: Payload transport, unmanned aerial vehicle (UAV), measurement system, path following controller, low-cost system.

Citation: D. C. Gandolfo, C. D. Rosales, L. R. Salinas, J. Gimenez, R. Carelli. Low-cost position and force measurement system for payload Transport using UAVs. International Journal of Automation and Computing, vol.18, no.4, pp.594-604, 2021. http://doi.org/10.1007/s11633-021-1281-4

\section{Introduction}

Currently, rotary-wing unmanned aerial vehicles (UAVs) are being widely used because of their great advantages for moving through hard-to-reach environment, as well as their abilities to take off and land in tight spaces. The problem of payload transport using these UAVs is having a great boom in the scientific community due to its countless potential applications, among which are: supply of medicines and food ${ }^{[1]}$, distribution of packages, assistance in different search and rescue scen$\operatorname{arios}^{[2]}$, water transport to fight forest fires, precise spraying in agriculture ${ }^{[3]}$, and transfer of construction materials. Quadrotor UAVs equipped with electric motors and fixed-pitch propellers became popular as a result of their compact size, low cost, and ability to operate safely in diverse environment while considering human presence. Furthermore, the mechanical composition of quadrotors are simpler than traditional helicopters ${ }^{[4]}$. These aerial vehicles have been widely used to transport payloads, and several aspects of the problem have been studied; involving one ${ }^{[5,6]}$ or more $\mathrm{UAVs}^{[7-10]}$, with the payload

\footnotetext{
Research Article

Manuscript received June 12, 2020; accepted January 22, 2021; published online April 13, 2021

Recommended by Associate Editor Jin-Hua She

Colored figures are available in the online version at https://link. springer.com/journal/11633

(C) The author (s) 2021
}

anchored to the $\mathrm{UAV}^{[11,12]}$ or hanging from cables ${ }^{[13]}$, using kinematic ${ }^{[14]}$ or dynamic ${ }^{[15]}$ controllers, with or without obstacle avoidance ${ }^{14]}$, among other considerations $^{[16]}$. There are companies dedicated to developing autonomous products for delivery systems and there are even companies that already offer UAVs designed to transport loads.

The payload-UAV link using cables is a considerable option since it can save time and energy, reduce weight, allow loading and unloading without landing, and reduce additional inertial effects $^{[17,18]}$. The difficulty of this transport problem is such that it has been addressed under restrictive assumptions such as navigation on a geometric plane ${ }^{[19-21]}$, or subdivided into three stages: takeoff, transport and unloading of the load ${ }^{[22]}$. The stability of a payload carried using cables is a key factor, and therefore, several alternatives have been studied ${ }^{[23-25]}$.

Most proposed controllers are based on the UAV position including schemes such as leader-follower ${ }^{[12]}$ for unifying commands in the cooperative transport. Another option is to control the system by directly defining desired references for the payload. The last option was adopted in [14] where two UAVs are controlled cooperatively so that the transported payload follows a desired trajectory while its weight is adequately distributed between the vehicles. This controller is based on permanent knowledge of the payload position and the force that it exerts on the UAVs, but the accurate estimation of the 
payload position in outdoor environments with low-cost equipment is a challenging problem. Attaching GPS or vision-based systems to perform the estimation implies extra costs that can be avoided in many situations. Besides, the estimation system must not be anchored to the payload because it will be delivered.

There are various systems for estimating the payload position in sensorized indoor environments $\left.{ }^{[15,} 26\right]$ which cannot be extrapolated to the outdoor environments usually required by the applications. Furthermore, most of these tracking systems are based on commercial motion capture devices, which are proprietary, expensive and not specially designed for UAVs ${ }^{[27]}$.

Another extremely important consideration is the force applied by the payload on the UAV, which is a key factor for knowing the cable stresses caused by the takeoff, excessive oscillations, or wind disturbances. This fact is even more critical in multi-UAV cooperation where the weight distribution should be diagrammed according to factors such as available energy or load capacity of the $\mathrm{UAVs}^{[14]}$. However, force sensors are generally expensive or have restrictive physical limitations such as extra weight or inadequate geometric arrangements for their use in UAVs.

Given these considerations, this paper presents a lowcost measurement system for loads transported using flexible cables, whose outputs are the payload position and the force exerted by it on a small-size UAV. The main qualities of the presented approach are the simplicity and low cost, compared to existing commercial ad-hoc sensors. Another important advantage is that this system does not need to be attached to the payload, allowing the load delivery system to be independent of the measurement system. Besides, this approach can be used in both outdoor and indoor environments.

This work also proposes a simple hardware structure that allows the real-time implementation of the proposal. The measurement system is experimentally validated both statically (mounting it on a static base) and dynamically (mounting it on a UAV to perform a path following mission in a real test scenario). For the dynamic validation, this work defines two kinematic path-following controllers based on the position of the UAV and the payload, respectively. Both controllers can be quickly adapted to any type of aircraft by only modifying an adaptation stage, avoiding the system identification required by dynamic controllers. Moreover, the controllers allow the incorporation of reference velocities modulated by the path curvature to bring significant performance improvements. An experimental analysis highlighting advantages and disadvantages of each controller with and without velocity modulation is incorporated. These experiments show the good performance of the developed measurement system and allow conclusions to be drawn about these options for payload transport using UAVs. Finally, this paper also includes a qualitative comparative analys- is with other payload measurement systems.

The paper is organized as follows. The measurement system and the hardware implementation are detailed in Section 2. The system validation is addressed in Section 3 . Experimental results are shown in Section 4. A comparative analysis with other measurement systems is presented in Section 5. Finally, conclusions are given in Section 6 .

\section{Low-cost measurement system}

This section describes the hardware used for the measuring system and explains how its parts are integrated. This system estimates the payload position in the body frame of the UAV using the angular displacements $\alpha$ and $\beta$ diagrammed in Fig. 1, where superscripts $B$ and $W$ refer to the body frame and the inertial (global) frame, respectively.

\subsection{Hardware}

The considered design requirements are low cost, reli-

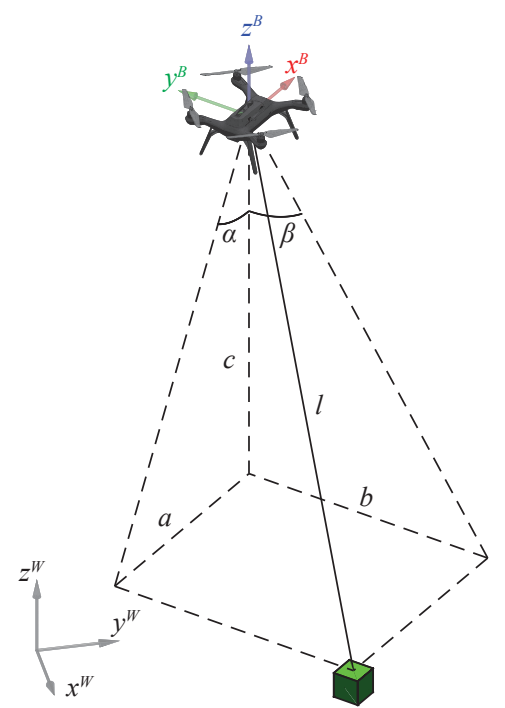

Fig. 1 Scheme of the load position in the body and global frames

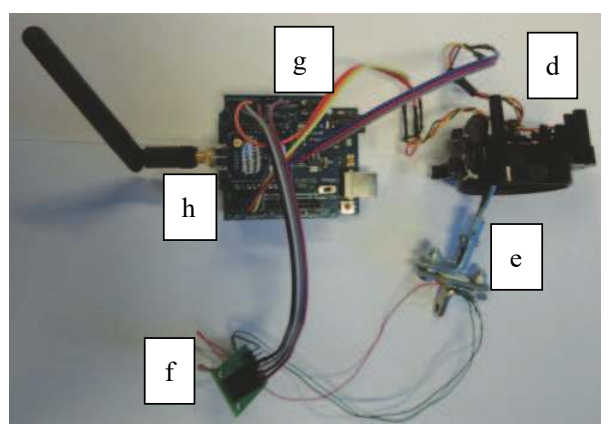

Fig. 2 Hardware of the measurement system. Potentiometers for (a) angle measurement, (b) load cell sensor, (c) module for HX711 load cells, (d) Arduino UNO, and (e) communication module. 
ability, robustness, and adaptability to outdoor/indoor environments. The proposed system shown in Fig. 2 has the following elements.

\subsubsection{Potentiometers for angle measurement}

It consists of two variable resistors placed in quadrature (one for the $x$-axis and another for the $y$-axis located perpendicularly). Because the mechanical arrangement of these resistors is extremely important, a Futaba radio control joystick is used to provide accurate and robust data. The maximum excursion allowed by this joystick on each axis is $30^{\circ}$.

\subsubsection{Load cell sensor}

The mechanism used to measure the force exerted by the payload was extracted from a low-scale commercial balance (The cost of this strain gauge is around $6 \$$ ). This alternative was chosen since the industrial load cells (with an appropriate shape and weight for this application) have a cost of around $500 \$$.

\subsubsection{Module for HX711 load cells}

This electronic board is a transmitter for load cells that has an integrated 24-bit $\mathrm{A} / \mathrm{D}$ converter, it is powered at $5 \mathrm{~V}$ and detects changes of $29 \mathrm{mV}$ in the measured signals. The module has four terminals to introduce information from the load cell, and the other four terminals for its connection with a microcontroller. Two of the later four terminals are for power and the other two for establishing an asynchronous communication with the microcontroller (data and clock).

\subsubsection{Arduino UNO}

An open-source microcontroller board equipped with sets of digital and analog input/output pins that may be interfaced to various expansion boards (shields) and other circuits. It has several advantages such as low cost, availability, simple and standard programming language, and multiple software libraries ready to use. Its characteristics are of public and wide knowledge.

\subsubsection{Communication module}

For the communication of the load position and force, an XBee PRO series 1 device of the Digi-Brand was used. These modules are suitable for this application because they are designed to perform wireless communications with high data traffic, low latency, and predictable synchronization. Furthermore, they work at $2.4 \mathrm{GHz}$, have great reach, and are easy to program through the XCTU software provided by the manufacturer. A point-to-multi- point type network was used, with acknowledgment and maximum header, 3 retries for the sending of each data and maximum power for transmission. This communication device was mounted directly on the Arduino development board through a dedicated shield for this purpose.

\subsection{Hardware integration}

This section briefly explains how the elements detailed above are interconnected to form the proposed measurement system (see Fig. 3). The angular displacements measured by the joystick potentiometers generate analogue signals, which are introduced into the microcontroller board for reading, digitization and processing. One end of the load cell is mechanically linked to the joystick lever (rigid connection that produces a joint movement between them), and the other end is linked to the payload through a cable. An interface HX711 is used to amplify the weak signals generated by the force sensor and to send the measurements to the microcontroller board. Once the necessary signals (two angles and a force) are introduced to the processing board, a $\mathrm{C}++$ program is used to signal conditioning and data transmission through the XBee PRO wireless communication device. These data are received by a station located on land (with another microcontroller platform and an XBee PRO), which introduce them in a $\mathrm{PC}$ through an USB port. Based on this information, the PC computes online the payload position in the body frame, and the force that it exerts on the UAV.

\subsection{Payload position estimation}

The UAV pose is given by its position $\xi^{W}=\left[x^{W}\right.$, $\left.y^{W}, z^{W}\right]^{\mathrm{T}}$ and its orientation $\sigma=[\psi, \theta, \varphi]^{\mathrm{T}}$ (pitch, roll and yaw, respectively), in a global reference system. Let $\xi_{\ell}^{B}=\left[x_{\ell}^{B}, y_{\ell}^{B}, z_{\ell}^{B}\right]^{\mathrm{T}}$ and $\xi_{\ell}^{W}=\left[x_{\ell}^{W}, y_{\ell}^{W}, z_{\ell}^{W}\right]^{\mathrm{T}}$ be the load position with respect to the body frame of the UAV and the global reference system, respectively (see Fig. 1). Then,

$$
\xi_{\ell}^{W}=\xi^{W}+R \xi_{\ell}^{B}
$$

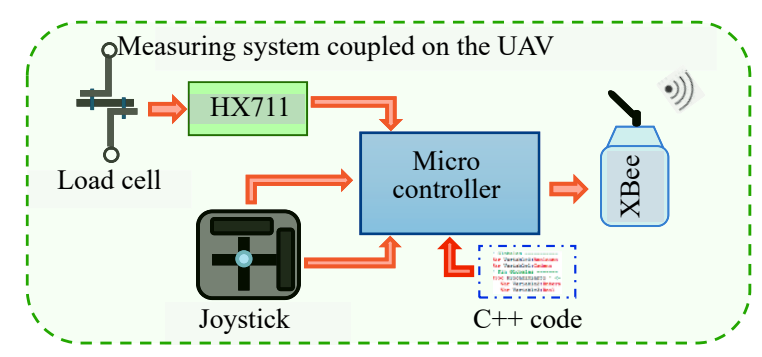

where $R=R_{\psi} R_{\theta} R_{\varphi}$ is the rotation matrix, with

Fig. 3 Scheme of the measurement system communication network to send and receive data 


$$
\begin{aligned}
& R_{\psi}=\left[\begin{array}{ccc}
\cos \psi & -\sin \psi & 0 \\
\sin \psi & \cos \psi & 0 \\
0 & 0 & 1
\end{array}\right] \\
& R_{\theta}=\left[\begin{array}{ccc}
\cos \theta & 0 & -\sin \theta \\
0 & 1 & 0 \\
\sin \theta & 0 & \cos \theta
\end{array}\right] \\
& R_{\varphi}=\left[\begin{array}{ccc}
1 & 0 & 0 \\
0 & \cos \varphi & -\sin \varphi \\
0 & \sin \varphi & \cos \varphi
\end{array}\right] .
\end{aligned}
$$

To estimate the load position, it is only necessary to know $\xi_{\ell}^{B}$, since $\xi^{W}$ and $R$ are provided by the on-board sensors of the UAV. This vehicle has a GPS and a redundant system with three IMUs (each has a gyroscope, accelerometer, magnetometer and barometer). All this sensory information is fused internally in the UAV with an extended Kalman filter to get the orientation, position and velocity data in a more robust and reliable way.

From the angles estimated by the system diagrammed in Fig. 1, if the cable is considered rigid and inelastic with constant length $\ell$, then

$$
\begin{gathered}
\left(x_{\ell}^{B}\right)^{2}+\left(y_{\ell}^{B}\right)^{2}+\left(z_{\ell}^{B}\right)^{2}=\ell^{2} \\
x_{\ell}^{B}=-z_{\ell}^{B} \tan \alpha \\
y_{\ell}^{B}=-z_{\ell}^{B} \tan \beta .
\end{gathered}
$$

Thus,

$$
\begin{aligned}
x_{\ell}^{B} & =\frac{\ell \tan \alpha}{\sqrt{1+\tan ^{2} \alpha+\tan ^{2} \beta}} \\
y_{\ell}^{B} & =\frac{\ell \tan \beta}{\sqrt{1+\tan ^{2} \alpha+\tan ^{2} \beta}} \\
z_{\ell}^{B} & =\frac{-\ell}{\sqrt{1+\tan ^{2} \alpha+\tan ^{2} \beta}} .
\end{aligned}
$$

\section{System validation}

This section tests the quality of the measurements made by the proposed system. Two validations, one static and the other dynamic, are performed to test the measuring system from complementary viewpoints. In static validation, the estimates of the proposed system are compared with the measurements of a highly tested reference system. On the other hand, in the dynamic validation, the system is mounted on a UAV and used to control it based on the obtained measurements.

\subsection{Static validation of the measurement system}

The designed measurement system must be compared with a known device in order to determine the accuracy and precision of the obtained measurements. This work uses the inertial measurement units (IMUs) of a 3DR SOLO as a validation system for the angles estimated with the proposed system. The structure shown in Fig. 4 was constructed to match the attitude estimations of the IMUs with the $\alpha$ and $\beta$ angles obtained by the measuring system. This additional platform allows us to keep the measurement system perpendicular to the $x-y$ plane of the inertial frame, and therefore, variations in pitch and roll angles coincide with the $\alpha$ and $\beta$ angles diagrammed in Fig. 1. Fig. 5 shows a comparative diagram of the measurements obtained while the UAV is randomly moved by hand.

Regarding the force measurements, several loads from $50 \mathrm{~g}$ to $1 \mathrm{~kg}$ were measured with the load cell to calibrate it. Based on these readings and using an interpolation process, a calibration curve is obtained. The estimated error is in the order of $6 \%$, which is sufficient for future research objectives where the load balance between UAVs is needed while a cooperative load transport is performed.

\subsection{Dynamic validation of the measure- ment system}

The measurement system is mounted on a multi-rotor

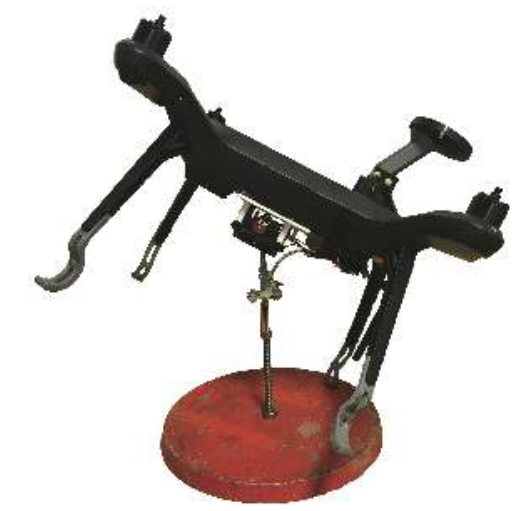

Fig. 4 System developed to perform the static validation of the measurement system
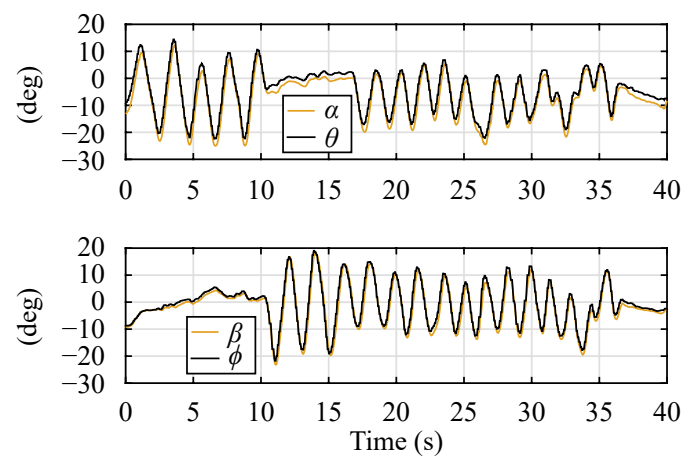

Fig. 5 IMUs' pitch $\theta$ and roll $\phi$ angles are shown in comparison with $\alpha$ and $\beta$ angles of the measurement system 
(SOLO model from 3D robotics) to dynamically validate it while the payload-UAV system follows a desired path. The section begins by describing the implemented pathfollowing controllers, which are based on the payload and UAV positions, respectively. Non-considered factors produce control errors in any real experimentation, these reference options allow us to distinguish the additional errors introduced by the proposed system. Subsequently, details of the system implementation on the commercial robot are given.

\subsubsection{Proposed controllers}

Two path controllers are used in this article, one is based on the UAV position $\eta=\xi^{W}$ (obtained by the onboard IMU estimations) and the other is based on the load position $\eta=\xi_{\ell}^{W}$ (obtained by the proposed methodology using (1) and (2)). For notational convenience, the desired path in both situations is denoted by $\eta^{*}=$ $\eta^{*}(s)=\left[x^{*}, y^{*}, z^{*}\right]^{\mathrm{T}}$. The angles

$$
\begin{aligned}
& \psi^{*}=\psi^{*}(s)=\operatorname{atan} 2\left(\frac{\mathrm{d} y^{*}}{\mathrm{~d} s}, \frac{\mathrm{d} x^{*}}{\mathrm{~d} s}\right) \\
& \theta^{*}=\theta^{*}(s)=\operatorname{atan} 2\left(\frac{\mathrm{d} z^{*}}{\mathrm{~d} s}, \sqrt{\left(\frac{\mathrm{d} x^{*}}{\mathrm{~d} s}\right)^{2}+\left(\frac{\mathrm{d} y^{*}}{\mathrm{~d} s}\right)^{2}}\right)
\end{aligned}
$$

characterize the orientation of the line tangent to the desired path. For each experiment realization, there is a function $s=s(t)$ given by

$$
s(t)=\arg \min _{s}\left\|\eta^{*}(s)-\eta(t)\right\|
$$

which characterizes the path point closest to the reference point $\eta$ at time $t$. That is, for each time $t, \eta^{*}(s(t))$ is the point on the desired path $\eta^{*}(s)$ closest to the reference point $\eta(t)$. This function varies from one experiment to another. The following error is defined by $\tilde{\eta}(t)=\eta^{*}(s(t))-\eta(t)$. In order to make $\tilde{\eta}(t) \rightarrow 0$ (pathfollowing error convergence), the kinematic controllers are defined by

$$
\eta_{c}=J^{-1}\left(v_{d}+K_{1} \tanh \left(K_{2} \tilde{\eta}\right)\right)
$$

where $K_{1}>0$ and $K_{2}>0$ are gain matrices,

$$
J=\left[\begin{array}{ccc}
\cos \psi & -\sin \psi & 0 \\
\sin \psi & \cos \psi & 0 \\
0 & 0 & 1
\end{array}\right]
$$

and $v_{d}=v_{d}\left[\cos \theta^{*} \cos \psi^{*}, \cos \theta^{*} \sin \psi^{*}, \sin \theta^{*}\right]^{\mathrm{T}} \quad$ is the desired velocity vector. Here, $v_{d}$ is the desired linear velocity, which can be a constant or variable. In this article, both cases are taken into account considering a velocity reduction scheme given by

$$
v_{d}=v_{d}(t)=\frac{v_{d, \max }}{1+k_{v_{d}, 1} \tanh \left(k_{v_{d}, 1} c(s(t)+\Delta)\right)}
$$

which depends on the path curvature $c(s(t)+\Delta)$ in a future path point indexed by $s(t)+\Delta, \Delta>0$, design constants $k_{v_{d}, 1}, k_{v_{d}, 2}>0$, and the maximum desired linear velocity $v_{d, \max }$.

On the other hand, to control yaw orientation, the following controller is used:

$$
\psi_{c}=\dot{\psi}^{*}(s(t))+k_{1, \psi} \tanh \left(k_{2, \psi}\left(\psi^{*}(s(t))-\psi(t)\right)\right)
$$

where $k_{1, \psi}, k_{2, \psi}>0$ are design constants.

\subsubsection{Implementation}

The topology shown in Fig. 6 is used to implement the controllers. The control algorithms were developed using Dronekit-Python API, allowing the communication with MAVLink protocol between the UAV and any application developed in Python. This provides access to telemetry information, status and parameters of the connected vehicle, and allows both mission management and direct control over the vehicle movements. The bidirectional UAV-PC communication is carried out through a WiFi network generated by the radio control of the UAV, to which the UAV is automatically connected unlike the PC that must be connected by hand. In each sampling period (set at $25 \mathrm{~ms}$ ), the UAV telemetry and the sensor status are received, while the control actions are sent contemplating the desired path and the tracking errors. The radio allows us to manually control the UAV if an eventual failure occurs, which is a key security component. On the other hand, the measurement system is connected to the PC through an USB port to receive the angular and force data (see Fig. 3). In this way, the measurement sys-

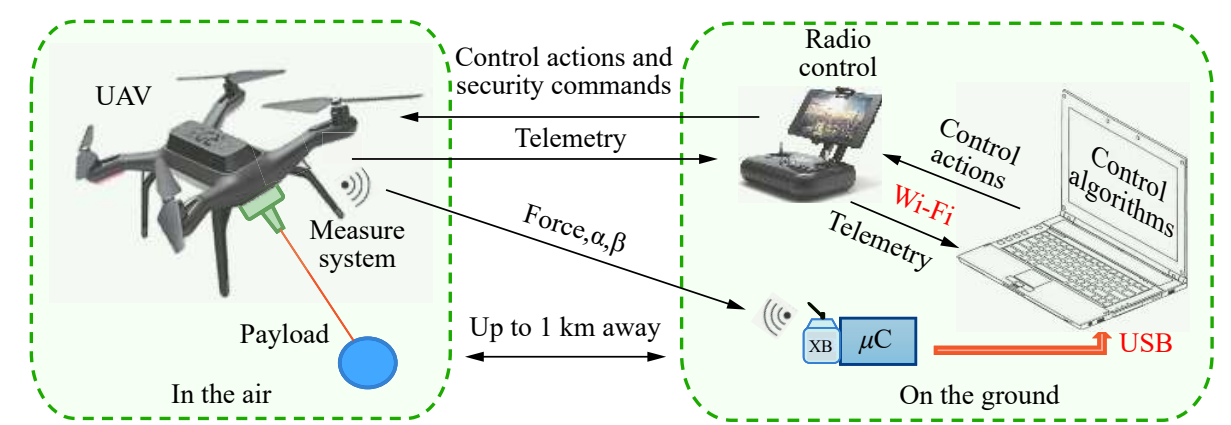

Fig. 6 Complete hardware architecture used in the experiments 
tem allows us to know the relative payload-UAV position during the flight execution.

\subsubsection{Dynamic validation}

This section presents the experiments of the controllers detailed in Section 3.2.1, which differ in that one is based on the UAV position and the other is based on the payload position. The first option is the most used in the literature, so a similar performance between these controllers implies a dynamic validation of the proposed measurement system.

The distance between the paths followed by the UAV and the payload should be approximately equal to the cable length. Besides, as the payload is transported by a single UAV, the projection of these paths on the $x-y$ plane should be similar if the UAV moves at low velocity and there are no external factors (such as wind) generating oscillations. However, these considerations begin to be false if the navigation velocity increases or undesired load oscillations appear, which would indicate that it is better to control based on the load position. On the other hand, reducing payload position errors is less straightforward than reducing tracking errors in the UAV position due to the sub-actuated characteristics of the system. Thus, there are two options for the payload to follow a desired path:

Option A: Define a desired path for the UAV raising the desired path for the payload a distance equal to the cable length, and follow it with the controller (3) based on the UAV position. This is the most common option in the literature.

Option B: Directly define a desired path for the payload, and follow it using the controller (3) based on the payload position estimated with the proposed system.

If the proposed system works correctly, then it should be similar to navigate under Option A or B. For comparative purposes, two experiments are performed considering the same reference paths and controller gains. The reference path is defined as a challenging eight-way shape at constant altitude to properly test the proposed system. In addition, the force exerted by the load in both cases is also monitored online, since the force oscillations reflect an unstable load transport.

The experimental results performed to validate the measuring platform are shown in Fig. 7. In Fig. 7(a), we present the $3 \mathrm{D}$ evolution for Option $\mathrm{A}$. The figure shows the reference and actual path followed by the UAV, as well as the path followed by the payload. Moreover, it includes the expected load path which is the projection of the UAV reference in the payload plane. Finally, Fig. 7(b) presents the results for Option B. The reference path for the payload, the path followed by the UAV, and the path followed by the payload are shown.

The difference between the payload path and the reference is presented in Fig. 8. The difference for Option A is calculated considering the projected UAV reference in the plane of the load. Thus, $\tilde{x}=x^{*}-x_{\ell}, \tilde{y}=y^{*}-y_{\ell}$, and

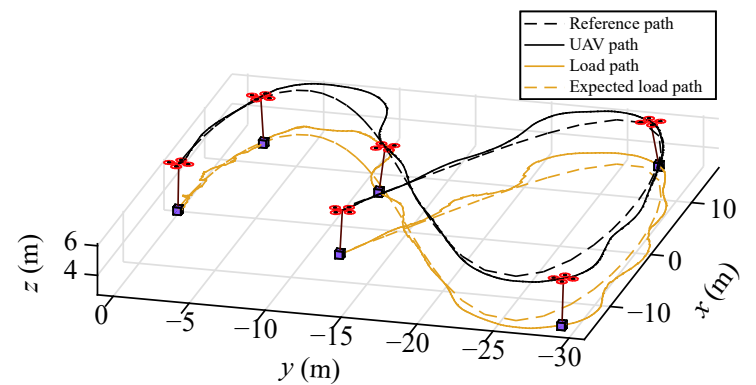

(a) Option A

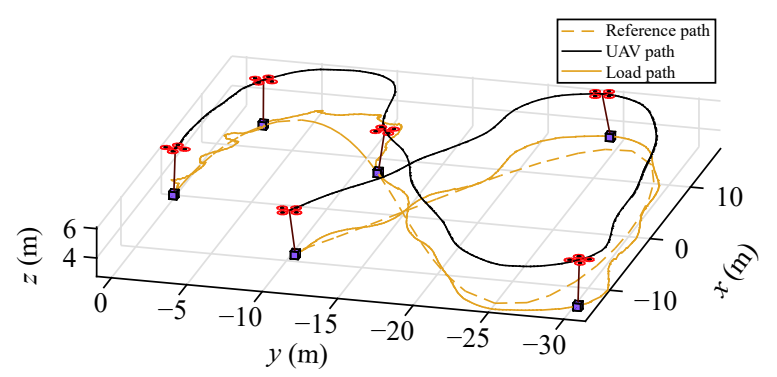

(b) Option B

Fig. 7 Dynamic validation
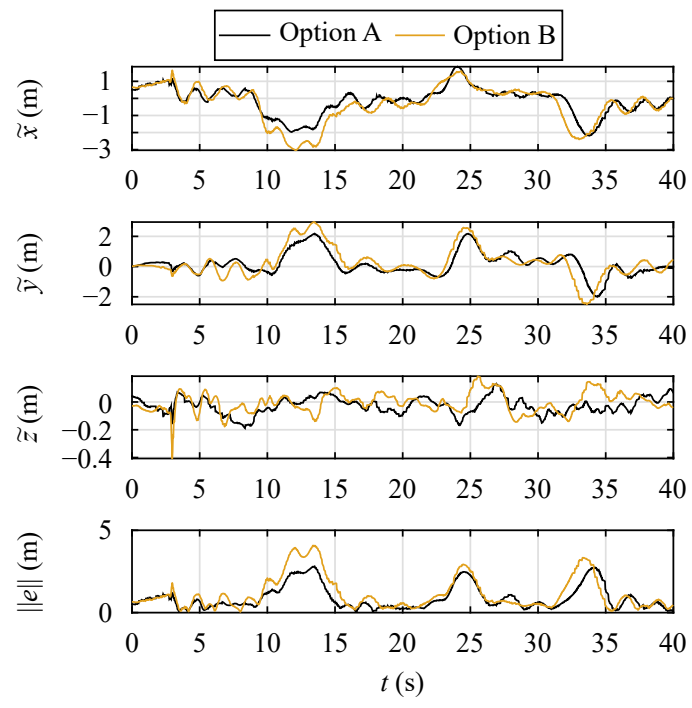

Fig. 8 Control error evolution

$\tilde{z}=z^{*}-z_{\ell}-\ell$. For Option $\mathrm{B}$, the difference and the control errors are calculated in the same way, because the reference path is defined over the load position. Therefore, $\tilde{x}=x^{*}-x_{\ell}, \tilde{y}=y^{*}-y_{\ell}$, and $\tilde{z}=z^{*}-z_{\ell}$. Besides, the norm of the error vector $\|e\|=\sqrt{\tilde{x}^{2}+\tilde{y}^{2}+\tilde{z}^{2}}$ is included with the objective of making a comparison between the two experiments, and it can be noted that both cases have similar errors.

Fig. 9 shows the evolution of the force exerted by the load and the angles $\alpha$ and $\beta$ for each experiment.

The payload used for the experiments has a weight of $220 \mathrm{~g}$ according to a precision balance. This value is approximately equal to the mean value measured by the system in stable state (see Fig. 9). The maximum effort 

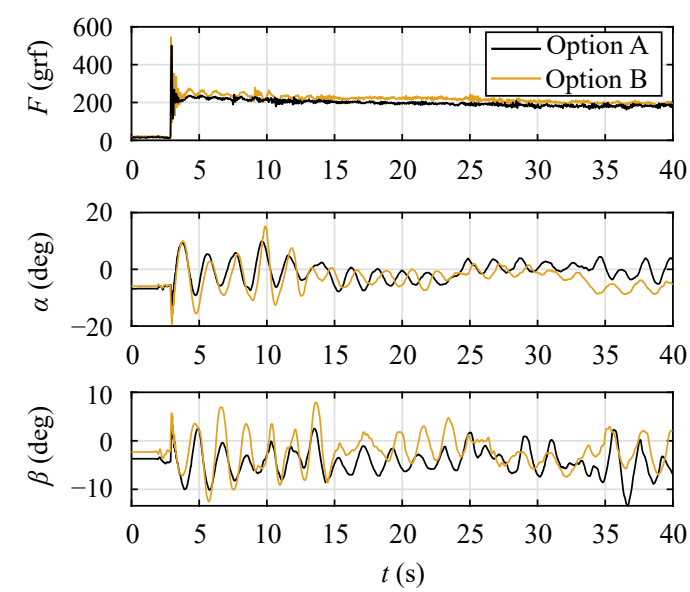

Fig. 9 Force exerted by the payload (measured by the load cell) and angles $\alpha$ and $\beta$ that allow estimating the payload position.

occurs at the take-off of the UAV, which corresponds to twice the payload weight. As can be seen in these preliminary experiments, the developed measuring system has a good dynamic behavior and the proposed hardware architecture exhibits a good performance.

\section{Experimental results and discussions}

In order to obtain conclusions regarding payload transport using a multirotor UAV, several experiments were conducted to compare the path followed by the payload in the four test scenarios described below:

Scenario 1: Control under Option A with fixed desired velocity $v_{d}$ set at $4 \mathrm{~m} / \mathrm{s}$.

Scenario 2: Control under Option B with fixed desired velocity $v_{d}$ set at $4 \mathrm{~m} / \mathrm{s}$.

Scenario 3: Control under Option A with desired velocity $v_{d}$ defined in function of the path curvature according to (4) with $v_{d, \max }=4 \mathrm{~m} / \mathrm{s}$.

Scenario 4: Control under Option B with desired velocity $v_{d}$ defined in function of the path curvature according to (4) with $v_{d, \max }=4 \mathrm{~m} / \mathrm{s}$.

In all scenarios, the reference path is an ellipse with a smaller radius of $20 \mathrm{~m}$ and a larger radius of $50 \mathrm{~m}$, located at a constant altitude of $5 \mathrm{~m}$. This curve is shown in a dotted black line in each plot of Fig. 10. The payload used for these experiments has a weight of $130 \mathrm{~g}$ (lower weight improve the autonomy of the UAV battery, and it is possible to perform a greater number of experiments). For each scenario, the control error in each axis is given in Fig.11, and the mean square error is presented in Fig. 12. In these graphs, the curve color determines to which scenario it corresponds according to the following criteria: Scenario 1: blue (dark gray); Scenario 2: green (gray); Scenario 3: gold (light gray); and Scenario 4: orange (medium dark gray). These colors are also used in Fig. 10 to show the evolution of the payload position, while the UAV position evolution is always plotted in
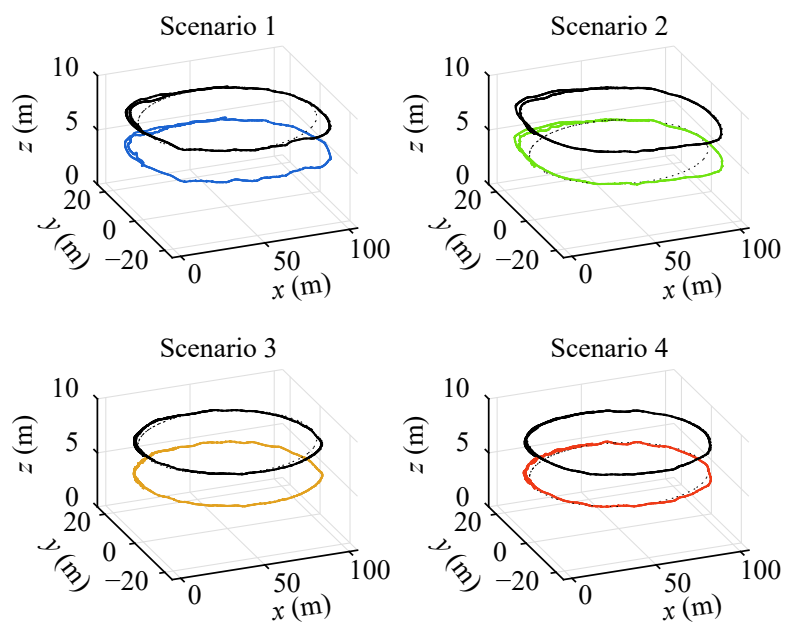

Fig. 10 UAV and payload position evolution for different test scenarios
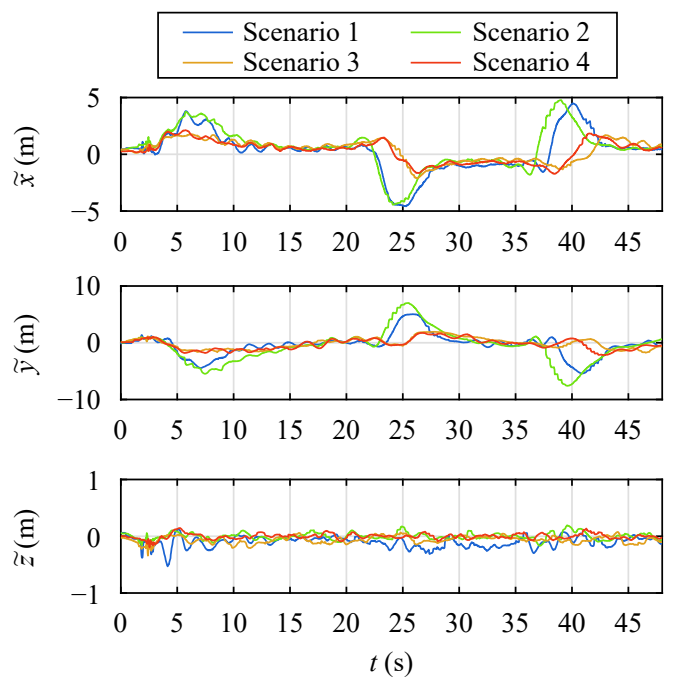

Fig. 11 Payload position error evolution for each scenario

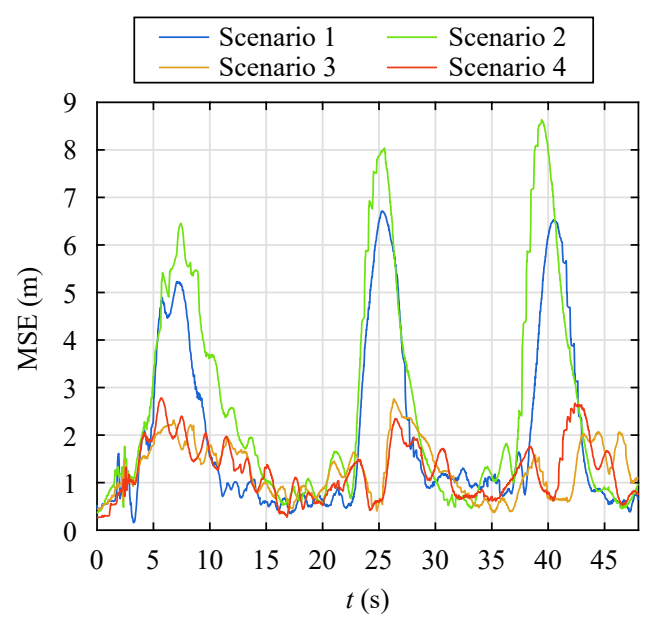

Fig. 12 Mean square error (MSE) of payload position for each scenario

black. The following link is a video of this experience: https://youtu.be/2QcfcdsdFIE. Note the improvement pro- 
duced by the proposed velocity reduction (Scenarios 3 and 4). Furthermore, the greatest errors are observed in the most curved zones of the desired reference path highlighting the need to incorporate control strategies with velocity modulated by geometric issues.

The $\alpha$ and $\beta$ angles for each scenario are plotted in Fig. 13 showing the payload oscillations during the transport task. On the other hand, Fig. 14 shows the drones' velocities, where the dashed black line represents the desired linear velocity, which is given by (4) for Scenarios 3 and 4.

Furthermore, the force exerted by the payload on the UAV for each scenario is shown in Fig. 15. During takeoff, the load exerts a downward force on the UAV ten times greater than the force exerted in a stable state. This peak in the force curve is not shown for scale reasons, but its
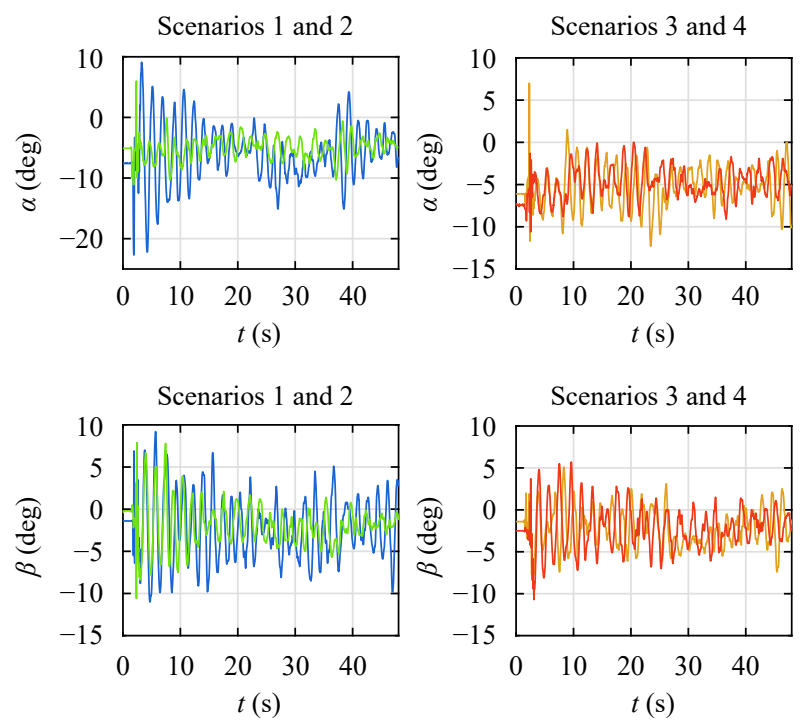

Fig. 13 Frontal $(\alpha)$ and lateral $(\beta)$ oscillation of payload for different scenarios
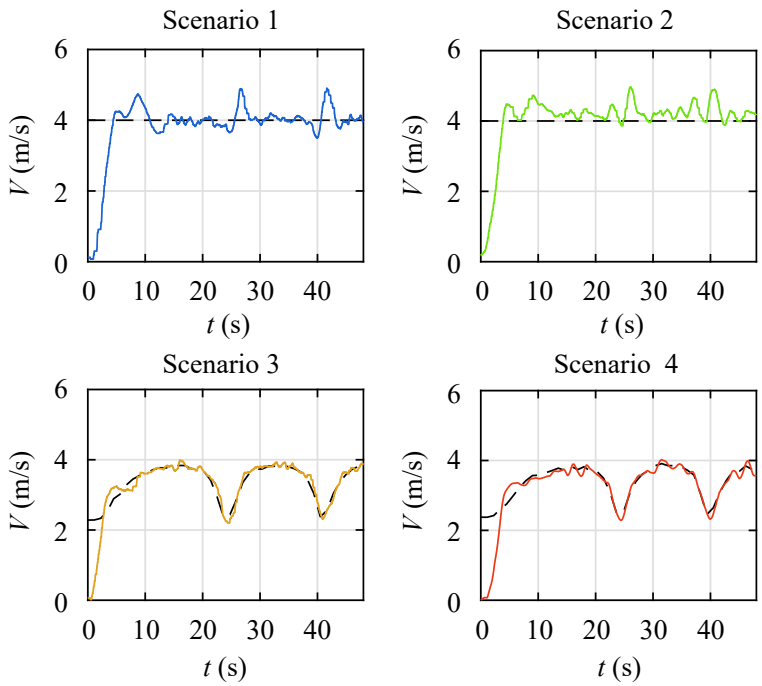

Fig. 14 Drone velocity along the path for different scenarios
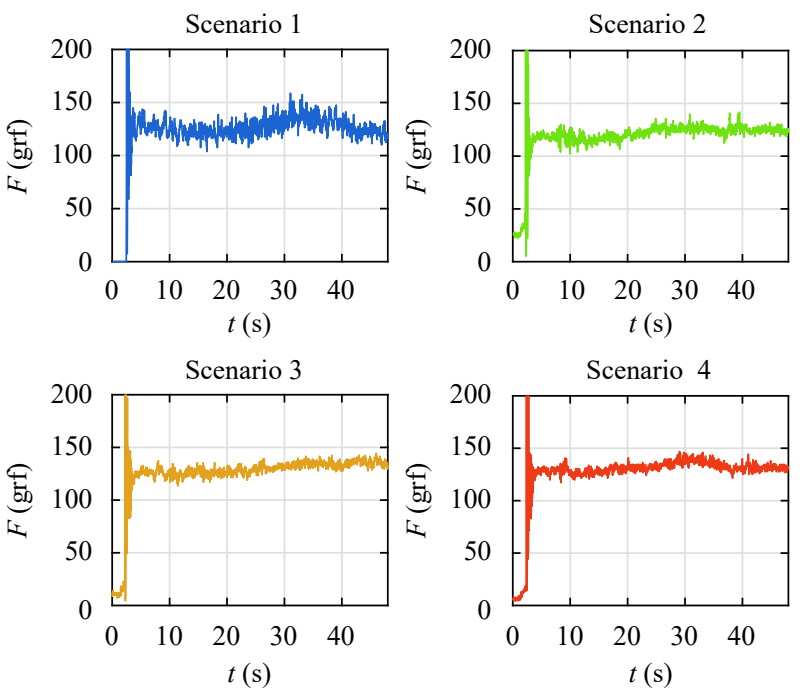

Fig. 15 Force exerted by the payload on the UAV for each scenario

magnitude is indicated in text.

The following observations can be highlighted from the experiments performed and data analysis:

1) Despite some differences in control errors, payload transport can be properly performed using the feedback considered in Options A and B.

2) Transporting the payload using Option A generates greater control errors in the most curved parts of the path (see Fig. 12) in the constant speed scenarios, but the oscillations of the payload are smaller (see plots for angles $\alpha$ and $\beta$ in Fig. 13). In certain applications, a low level of oscillations may be more important than smaller control errors, which makes scenario 2 better than scenario 1 (feedback the payload position brings advantages in this regard). Thus, the more convenient approach should be chosen according to the application.

3) The path curvature affects control errors and load oscillations, and thus, it should be incorporated as a key factor in any comparison study. Navigation at low speed can mitigate this problem, but this entails a higher energy consumption (since the aircraft remains in the air longer, maintaining the transported weight). A compromise solution between these situations is the velocity modulation according to geometric factors, which directly impacts on tracking errors, payload oscillations, and energy consumption. These considerations makes the speed modulation one of the most attractive alternatives.

4) The force exerted by the payload is variable reaching a short-time peak nine times greater than the load weight at takeoff. This situation must be considered when choosing the UAV with which a certain payload will be transported. Besides, the control strategy could produce a smooth takeoff that allows the payload to be lifted gradually and without a brisk jerk. This consideration will be taken into account in future works.

5) The control strategy proposed here is based on a 
kinematic model of the UAV. Its great virtue is the simplicity and flexibility to be adapted to other types of aircraft. However, dynamic offsets of the UAV-payload system can be taken into account to achieve a better performance. This type of compensation is under study and will be considered in a future work.

\section{Comparative analysis with other payload measurement systems}

As mentioned before, an increasing number of research groups have been studying payload transport systems using one or multiple UAVs, especially in the last ten years. Focusing in the studies with experimental results, a qualitative comparison is presented in Table 1. The payload measurement system of each study is analyzed in six topics: position sensing, force sensing, use of motion capture systems, environment, cost and weight. The position sensing is "relative" (local) when the load is measured with information provided by on-board sensors. It has the great advantage of not needing external sensors but the load position depends on the vehicle position, and hence is affected by the measurement errors of the latter. Our proposed system, along with $[2,13]$, has this type of sensing and uses the same principle to measure the load position, with some differences; Bernad et al.[2] use magnetic encoders and Takahashi et al.[13] use a multi-axis force/torque sensor to find out the cable (sling) angles. Instead, local sensing in [8] is based on visual tracking using a fish-eye lens camera, however, only slow motion in an indoor environment were tested. When the position sensing is "absolute" (global), the external sensors usually need an infrastructure, restricting the experiments to indoor or small-size outdoor environments. Moreover, all the studies with this type of measurement analyzed here use very expensive indoor motion capture systems. The force exerted by the load on the UAV is only measured in $[2,13]$ and our proposal, nonetheless, our system is smaller, lighter and cost much less than the other two systems. Some studies did not measure the load position/force but estimated them from the state of the vehicles using a geometric or dynamic model-based algorithm.

\section{Conclusions}

In this work, a low-cost system is developed to measure the payload position and the force exerted by it on the UAV. System validations are performed statically and dynamically. For the first validation, the angles estimated by the system are tested using the on-board IMUs, and the estimated weight is compared with the measurements obtained with precision balances. For the second validation, path controllers based on payload position and UAV position are compared to verify if the measurement system can be used efficiently in real applications. This system will allow controlling a UAV team to cooperatively transport a payload considering key factors such as unified commands, adequate weight distribution among vehicles, and reduction of load oscillations.

The dynamic validation of the system allows us to draw several useful conclusions in order to guide future research. In the different control test scenarios, navigation was performed correctly using both the payload position and UAV position feedbacks. On the one hand, fewer tracking errors and higher payload oscillations are obtained when using UAV position feedback. Then, the choice of one scenario or another depends on the demands of the particular task. On the other hand, the best

Table 1 Qualitative comparison with other payload measurement systems in the literature

\begin{tabular}{|c|c|c|c|c|c|c|}
\hline & Position sensing & Force sensing & $\begin{array}{c}\text { Motion capture } \\
\text { system }\end{array}$ & Environment & Cost & Weight \\
\hline Our system & Relative & Yes & No & Outdoor & Very low & Low \\
\hline$[2]$ & Relative & Yes & No & Outdoor & Medium & Medium \\
\hline$[5]$ & Absolute & No & Yes & Indoor & Very high & Very low \\
\hline$[6]$ & No* & No & Yes & Indoor & Very high & None \\
\hline$[7]$ & $\mathrm{No}^{*}$ & No & Yes & Indoor & Very high & None \\
\hline$[8]$ & Relative & No & $\mathrm{No}^{\dagger}$ & Indoor & Low & Very low \\
\hline$[9]$ & $\mathrm{No}^{*}$ & No & Yes & Indoor & Very high & None \\
\hline$[10]$ & Absolute & No & Yes & Indoor & Very high & Very low \\
\hline [13] & Relative & Yes & No & Outdoor & High & High \\
\hline$[15]$ & Absolute & No & Yes & Indoor & Very high & Very low \\
\hline$[16]$ & No & No* & No & Indoor & Very low & Very low \\
\hline$[17]$ & Absolute & No & Yes & Indoor & Very high & Very low \\
\hline [18] & Absolute & No & Yes & Indoor & Very high & Very low \\
\hline
\end{tabular}

* The position/force is not measured but estimated from the state of the vehicles. $\dagger$ A motion capture system is used but only as a validation tool. 
results (in terms of tracking errors and oscillations) are obtained in scenarios with speed reductions based on geometric issues. Considering these experiments, the use of control strategies that modulate the transport speed is recommended to obtain a better cost-benefit ratio between tracking errors, oscillations, and energy consumption. Besides, the forces the UAV is subjected to at takeoff are much greater than the weight of the payload, so a smooth takeoff should be included in the designed control strategy. Finally, a dynamic compensation could improve the performance of the proposed strategies.

\section{Acknowledgments}

This research was supported by National Scientific and Technical Research Council (CONICET) and the National University of San Juan (UNSJ), both from Argentina.

\section{Open access}

This article is licensed under a Creative Commons Attribution 4.0 International License, which permits use, sharing, adaptation, distribution and reproduction in any medium or format, as long as you give appropriate credit to the original author(s) and the source, provide a link to the Creative Commons licence, and indicate if changes were made.

The images or other third party material in this article are included in the article's Creative Commons licence, unless indicated otherwise in a credit line to the material. If material is not included in the article's Creative Commons licence and your intended use is not permitted by statutory regulation or exceeds the permitted use, you will need to obtain permission directly from the copyright holder.

To view a copy of this licence, visit http://creativecommons.org/licenses/by/4.0/.

\section{References}

[1] A. Gupta, A. Singh, D. Bharadwaj, A. K. Mondal. Humans and robots: A mutually inclusive relationship in a contagious world. International Journal of Automation and Computing, published online. DOI: 10.1007/s11633020-1266-8.

[2] M. Bernard, K. Kondak, I. Maza, A. Ollero. Autonomous transportation and deployment with aerial robots for search and rescue missions. Journal of Field Robotics, vol. 28, no. 6, pp. 914-931, 2011. DOI: 10.1002/rob.20401.

[3] U. M. Rao Mogili, B. B. V. L. Deepak. Review on application of drone systems in precision agriculture. Procedia Computer Science, vol.133, pp. 502-509, 2018. DOI: 10.10 16/j.procs.2018.07.063.

[4] D. C. Gandolfo, L. R. Salinas, A. Brandão, J. M. Toibero. Stable path-following control for a quadrotor helicopter considering energy consumption. IEEE Transactions on Control Systems Technology, vol. 25, no. 4, pp.1423-1430, 2017. DOI: 10.1109/TCST.2016.2601288.
[5] K. Sreenath, N. Michael, V. Kumar. Trajectory generation and control of a quadrotor with a cable-suspended load-a differentially-flat hybrid system. In Proceedings of IEEE International Conference on Robotics and Automation, IEEE, Karlsruhe, Germany, pp.4888-4895, 2013. DOI: 10.1109/ICRA.2013.6631275.

[6] F. A. Goodarzi, D. Lee, T. Lee. Geometric control of a quadrotor UAV transporting a payload connected via flexible cable. International Journal of Control, Automation and Systems, vol.13, no.6, pp.1486-1498, 2015. DOI: 10.1007/s12555-014-0304-0.

[7] N. Michael, J. Fink, V. Kumar. Cooperative manipulation and transportation with aerial robots. Autonomous Robots, vol.30, no.1, pp.73-86, 2011. DOI: $10.1007 /$ s10514010-9205-0.

[8] M. Gassner, T. Cieslewski, D. Scaramuzza. Dynamic collaboration without communication: Vision-based cablesuspended load transport with two quadrotors. In Proceedings of IEEE International Conference on Robotics and Automation, IEEE, Singapore, pp.5196-5202, 2017. DOI: 10.1109/ICRA.2017.7989609.

[9] H. G. De Marina, E. Smeur. Flexible collaborative transportation by a team of rotorcraft. In Proceedings of 2019 International Conference on Robotics and Automation, IEEE, Montreal, Canada, pp.1074-1080, 2019. DOI: 10.1109/ICRA.2019.8794316.

[10] K. K. Dhiman, M. Kothari, A. Abhishek. Autonomous load control and transportation using multiple quadrotors. Journal of Aerospace Information Systems, vol.17, no. 8, pp. 417-435, 2020. DOI: 10.2514/1.I010787.

[11] F. Ruggiero, V. Lippiello, A. Ollero. Aerial manipulation: A literature review. IEEE Robotics and Automation Letters, vol.3, no.3, pp.1957-1964, 2018. DOI: 10.1109/LRA. 2018.2808541.

[12] Y. C. Paw, G. J. Balas. Development and application of an integrated framework for small UAV flight control development. Mechatronics, vol.21, no.5, pp.789-802, 2011. DOI: 10.1016/j.mechatronics.2010.09.009.

[13] M. D. Takahashi, M. S. Whalley, M. G. Berrios, G. J. Schulein. Flight validation of a system for autonomous rotorcraft multilift. Journal of the American Helicopter Society, vol.64, no.3, pp.1-13, 2019. DOI: 10.4050/JAHS.64. 032001.

[14] J. Gimenez, D. C. Gandolfo, L. R. Salinas, C. Rosales, R. Carelli. Multi-objective control for cooperative payload transport with rotorcraft UAVs. ISA Transactions, vol. 80, pp. 491-502, 2018. DOI: 10.1016/j.isatra.2018.05.022.

[15] X. Liang, Y. C. Fang, N. Sun, H. Lin. A novel energycoupling-based hierarchical control approach for unmanned quadrotor transportation systems. IEEE/ASME Transactions on Mechatronics, vol.24, no.1, pp.248-259, 2019. DOI: 10.1109/TMECH.2019.2891083.

[16] A. Tagliabue, M. Kamel, S. Verling, R. Siegwart, J. Nieto. Collaborative transportation using MAVs via passive force control. In Proceedings of International Conference on Robotics and Automation, IEEE, Singapore, pp.5766-5773, 2017. DOI: 10.1109/ICRA.2017.7989678.

[17] I. Palunko, P. Cruz, R. Fierro. Agile load transportation: Safe and efficient load manipulation with aerial robots. IEEE Robotics \& Automation Magazine, vol.19, no.3, pp. 69-79, 2012. DOI: 10.1109/MRA.2012.2205617.

[18] P. Foehn, D. Falanga, N. Kuppuswamy, R. Tedrake, D. Scaramuzza. Fast trajectory optimization for agile quadro- 
tor maneuvers with a cable-suspended payload. In Proceedings of Robotics: Science and Systems, Boston, USA, 2017.

[19] S. Tang. Aggressive Maneuvering of a Quadrotor with a Cable-Suspended Payload, Ph. D. dissertation, Department of Mechanical Engineering and Applied Mechanics, University of Pennsylvania, USA, 2014.

[20] J. J. Potter, C. J. Adams, W. Singhose. A planar experimental remote-controlled helicopter with a suspended load. IEEE/ASME Transactions on Mechatronics, vol. 20, no. 5, pp. 2496-2503, 2015. DOI: 10.1109/TMECH.2014.238 6801.

[21] I. H. B. Pizetta, A. S. Brandão, M. Sarcinelli-Filho. Cooperative quadrotors carrying a suspended load. In Proceedings of International Conference on Unmanned Aircraft Systems, IEEE, Arlington, USA, pp. 1049-1055, 2016. DOI: 10.1109/ICUAS.2016.7502605.

[22] P. J. Cruz, R. Fierro. Cable-suspended load lifting by a quadrotor UAV: Hybrid model, trajectory generation, and control. Autonomous Robots, vol.41, no.8, pp. 1629-1643, 2017. DOI: $10.1007 / \mathrm{s} 10514-017-9632-2$.

[23] S. C. Dai, T. Lee, D. S. Bernstein. Adaptive control of a quadrotor UAV transporting a cable-suspended load with unknown mass. In Proceedings of the 53rd IEEE Conference on Decision and Control, IEEE, Los Angeles, USA, pp. 6149-6154, 2014. DOI: 10.1109/CDC.2014.7040352.

[24] M. E. Guerrero, D. A. Mercado, R. Lozano, C. D. García. Passivity based control for a quadrotor UAV transporting a cable-suspended payload with minimum swing. In Proceedings of the 54th IEEE Conference on Decision and Control, IEEE, Osaka, Japan, pp.6718-6723, 2015. DOI: 10.1109/CDC.2015.7403277.

[25] G. V. Raffo, M. M. de Almeida. Nonlinear robust control of a quadrotor UAV for load transportation with swing improvement. In Proceedings of IEEE American Control Conference, IEEE, Boston, USA, pp.3156-3162, 2016. DOI: 10.1109/ACC.2016.7525403.

[26] S. Tang, V. Kumar. Mixed integer quadratic program trajectory generation for a quadrotor with a cable-suspended payload. In Proceedings of IEEE International Conference on Robotics and Automation, IEEE, Seattle, USA, pp. 2216-2222, 2015. DOI: 10.1109/ICRA.2015.7139492.

[27] Q. Fu, X. Y. Chen, W. He. A survey on 3D visual tracking of multicopters. International Journal of Automation and Computing, vol.16, no.6, pp.707-719, 2019. DOI: 10.10 07/s11633-019-1199-2.

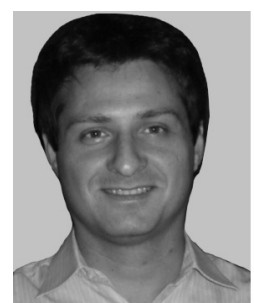

Daniel Ceferino Gandolfo received the B. Eng. degree in electronic engineering from National University of San Juan (UNSJ), Argentina in 2006. He has been working as automation engineer in the industry until 2009 and received the Ph.D. degree in control systems engineering from UNSJ, Argentina in 2014. Currently, he is a researcher of Argentinean National Council for Scientific Research, and an associate professor in Institute of Automatics, UNSJ, Argentina.

His research interests include algorithms for management energy systems and optimal control strategies with application in unmanned aerial vehicles.

E-mail: dgandolfo@inaut.unsj.edu.ar (Corresponding author)

\section{ORCID iD: 0000-0002-4938-2105}

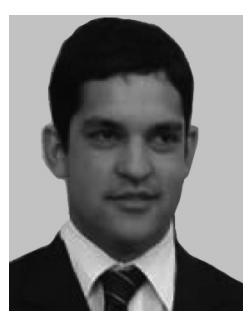

Claudio D. Rosales received the B. Eng. degree in electronic engineer from National University of San Juan, Argentine in 2009, and the Ph.D. degree in control systems engineering from UNSJ, Argentina in 2014, and the Ph.D. degree in electric engineering from the Federal University of Espírito Santo, Brazil, in 2018. Currently, he is an assistant researcher of the Council for Scientific and Technological Research, Argentina, and an associate professor in the Institute of Automatic, UNSJ.

His research interests included algorithms for multi-robot systems, nonlinear control, artificial intelligence, and aerial robotic.

E-mail: crosales@inaut.unsj.edu.ar

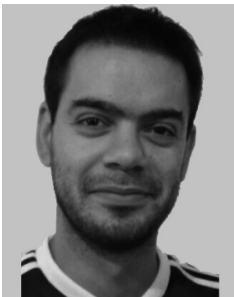

Lucio R. Salinas received the B.Eng. degree in electronic engineering and the $\mathrm{Ph} . \mathrm{D}$. degree in control systems engineering from National University of San Juan, Argentina in 2008 and 2013, respectively. $\mathrm{He}$ is an associate researcher at National Scientific and Technical Research Council (CONICET) and an assistant professor at Institue of Automation, UNSJ.

His research interests include robotics, teleoperation systems, unmanned aerial vehicles, human-machine systems and software development.

E-mail: lsalinas@inaut.unsj.edu.ar

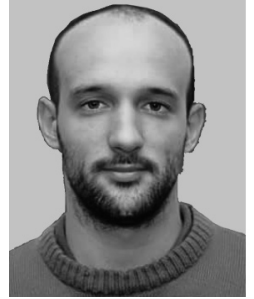

Argentina.

His research interests include probabilistic and statistical implementations of robotics, such as SLAM algorithms.

E-mail: jgimenez@inaut.unsj.edu.ar

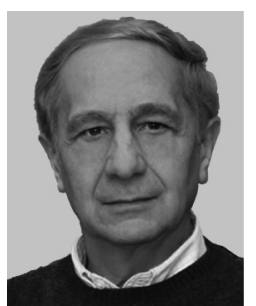

Ricardo Carelli received E. Eng. degree in engineering from the National University of San Juan, Argentina in 1976, and received the $\mathrm{Ph}$. D. degree in electrical engineering from National University of Mexico (UNAM), Mexico in 1989. He is a professor at National University of San Juan, Argentina and a senior researcher by contract with National Council for Scientific and Technical Research, Argentina. He has been the Director of the Institute of Automation, National University of San Juan, Argentina from 2008 to 2019. He has published more than a hundred scientific articles en indexed journals on control and robotics.

His research interests include robotics, manufacturing systems, adaptive control and artificial intelligence applied to automatic control.

E-mail: rcarelli@inaut.unsj.edu.ar 